\title{
Sarcopenia is associated with hypertension in older adults: a systematic review and meta-analysis
}

\author{
Tingting Bai ${ }^{\dagger}$, Fang Fang ${ }^{\dagger}$, Feika $\mathrm{Li}^{\dagger}$, Yan Ren, Jiaan Hu and Jiumei Cao
}

\begin{abstract}
Background: Sarcopenia, particularly low handgrip strength has been observed and correlated in association with hypertension among the older people. However, the results reported in different studies were inconsistent. In the current study, we conducted a systematic review and meta-analysis to reveal the significant association between sarcopenia, handgrip strength, and hypertension in older adults.

Methods: PubMed, MEDLINE, Cochrane Library, and EMBASE databases were searched from inception to 15 November 2019 to retrieve the original research studies that addressed the association between sarcopenia, handgrip strength, and hypertension. All the relevant data were retrieved, analyzed, and summarized.

Results: Twelve articles met the inclusion criteria and a total of 21,301 participants were included in the metaanalysis. Eight eligible studies have reported the odd ratios (ORs) of hypertension and sarcopenia, and the ORs ranged from 0.41 to 4.38 . When pooled the ORs together, the summarized OR was 1.29 [95\% confidence interval $(\mathrm{Cl})=1.00-1.67]$. The summarized $\mathrm{OR}$ for the Asian group $1.50(95 \% \mathrm{Cl}=1.35-1.67)$ was significantly higher than that of Caucasian group $1.08(95 \% \mathrm{Cl}=0.39-2.97)$. Eleven studies have provided the data on association between handgrip strength and hypertension. The overall OR and $95 \% \mathrm{Cl}$ was $0.99(95 \% \mathrm{Cl}=0.80-1.23)$, showing no significant association.

Conclusion: Sarcopenia was associated with hypertension, but no correlation was found between handgrip strength and hypertension in older adults.
\end{abstract}

Keywords: Sarcopenia, Handgrip strength, Hypertension, Older adults, Meta-analysis

\section{Background}

The number of elderly people among the world population is expected to reach about $30 \%$ by the year 2050 [1]. However, the aging process is accompanied with alterations in some physiological systems leading to the development of geriatric syndromes and chronic diseases. It was previously reported that hypertension affects more than $70 \%$ of older people [2] and

\footnotetext{
* Correspondence: cjm11261@rjh.com.cn

${ }^{\dagger}$ Tingting Bai, Fang Fang and Feika Li contributed equally to this work. Department of Geriatrics, Ruijin Hospital, Shanghai Jiaotong University School of Medicine, Shanghai, China
}

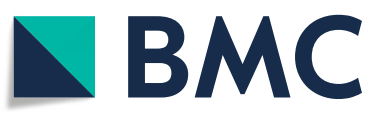

(C) The Author(s). 2020 Open Access This article is licensed under a Creative Commons Attribution 4.0 International License, which permits use, sharing, adaptation, distribution and reproduction in any medium or format, as long as you give appropriate credit to the original author(s) and the source, provide a link to the Creative Commons licence, and indicate if changes were made. The images or other third party material in this article are included in the article's Creative Commons licence, unless indicated otherwise in a credit line to the material. If material is not included in the article's Creative Commons licence and your intended use is not permitted by statutory regulation or exceeds the permitted use, you will need to obtain permission directly from the copyright holder. To view a copy of this licence, visit http://creativecommons.org/licenses/by/4.0/. The Creative Commons Public Domain Dedication waiver (http://creativecommons.org/publicdomain/zero/1.0/) applies to the data made available in this article, unless otherwise stated in a credit line to the data. predisposes them to an increased risk of stroke (i.e., hemorrhagic and ischemic) and myocardial infarction [2, 3]. In the past few years, a large number of studies have indicated that hypertension is predominantly associated with elevated cardiovascular risk [4-6].

Recently, data from population studies have demonstrated that sarcopenia, a neuromuscular disease characterized by a progressive muscular atrophy accompanied by diminished muscle strength and/or lower muscle limb function, could be associated with with hypertension [7-9]. The consensus definitions of sarcopenia by European geriatric and gerontological societies proposed 
a mandatory measurement of handgrip strength to diagnose probable sarcopenia [10]. Meanwhile, sarcopenia has been demonstrated to have substantial associations with the aging process and can lead to significant morbidity and disability, including the loss of independence, poor quality of life, and mortality [11-14]. Sarcopenia has been reported to be associated with several contributing factors, such as primarily advanced age, immobility, inadequate nutrition, neurodegenerative disease, malignancy, chronic multiple endocrine disorders, and cardiometabolic disease [12]. Moreover, the rate of sarcopenia in the elderly is expected to increase in the future [15] and is becoming a major public health problem [16].

The handgrip strength examination is often applied as a diagnostic approach for sarcopenia in clinical settings, and such a measure is considered inexpensive, simple, easy, and can be implemented with a portable measuring tool. Until now, a significant association between sarcopenia and grip strength, and hypertension in older adults was controversial $[7-9,17,18]$ and has not been systemically summarized. Therefore, a comprehensive investigation on this topic might allow an early detection of the key risk factors of hypertension in elderly patients suffering with sarcopenia and may help to effectively organize prevention or treatment strategies associated with specific vulnerability factors.

\section{Methods}

\section{Literature search}

A literature search was conducted using the individual and joint keywords, "handgrip strength", "grip strength", "sarcopenia", and "hypertension" following the Preferred Reporting Items for Systematic Reviews and MetaAnalyses (PRISMA) statement [19]. To enhance the retrieval of potential literatures, we kept the search terms as broad as possible to identify the relevant publications. A systematic electronic data search of PubMed, MEDL INE, Cochrane Library, and EMBASE databases were performed up to 15 November 2019. Moreover, the bibliographies of all relevant studies and reviews, and Google Scholar for literature citing relevant studies were also checked and identified.

\section{Eligibility criteria}

The inclusion criteria were as follows: (1) observational studies addressing the association between sarcopenia and hypertension, or handgrip strength and hypertension; (2) studies providing clear diagnostic criteria of sarcopenia and hypertension; (3) necessary data extracted from original studies; (4) studies published in English; and (5) only the study providing more detailed information was included if the population was reported in duplicate.
Reviews, case reports, abstracts or posters for conferences, studies focused on animal experiments or experiments in vitro, and studies published in languages other than English or Chinese were excluded.

\section{Data extraction}

Two investigators (TTB and FF) have independently extracted the necessary information of included studies using a customized and standardized form, and the consensus were reached on all items by these two authors. For each included study, the following information were extracted: the author and year of publication, country, study design, sample size, patient demographic characteristics (e.g., age, sex, and nation), diagnostic criteria of sarcopenia and hypertension, sample size and characteristics for each group, follow-up period, and outcomes of each group.

\section{Quality scoring of studies}

Two reviewers (FKL and JMC) have assessed the methodological strength of included studies independently in order to interpret the validity of any findings observed through the Newcastle-Ottawa Scale (NOS), a procedure performed to independently assess the methodological quality of meta-analysis of observational studies [20]. Newcastle-Ottawa Scale included three categories of factors: (1) patient selection (three items); (2) comparability of the two study arms (two items); and (3) assessment of the outcomes (two items). The detailed criteria for the three assessments are: whether the cases were defined adequately, the representativeness of the cases, the process of selection and definition of controls, comparability of cases and controls based on the design or analysis, ascertainment of exposure, the same method of ascertainment for cases and controls, and nonresponse rate.

Studies were awarded one star for each numbered item within the selection and exposure categories, and one or two stars for comparability. Studies were graded on an ordinal scoring scale. The scores ranged from 2 to 9 stars. Therefore, a scale of 0 to 4 stars was considered as poor quality, 5 to 6 stars as moderate quality, and 7 to 9 stars as high quality.

\section{Statistical analysis}

The inverse variance method with random effects was conducted to summarize the dichotomous outcomes, odd ratios (ORs), and 95\% confidence intervals (CIs). Stratified analyses were also performed with respect to the characteristics of the study population and outcome. Heterogeneity between included studies was assessed using the $I^{2}$ and $\mathrm{Q}$ tests. Heterogeneity was defined as low, moderate, and high to $\mathrm{I}^{2}$ values of 25,50 , and $75 \%$, respectively [21]. The Begg rank correlation [22] and 
Egger weighted regression methods [23] were used to assess the publication bias $(P<0.05$ was considered indicative of a statistically significant publication bias). Review Manager (Version 5.3, The Cochrane Collaboration, Oxford, UK) was used for generation of forest plots and statistical analyses. The Begg and Egger tests were assessed by STATA 15.0 (Stata Corporation, College Station, TX, USA). A $P$ value of $<0.05$ was considered significant for all analysis.

\section{Results}

\section{Study selection}

In total, 1221 studies were retrieved as potentially relevant literature reports through the initial searches in different databases, after removing the duplicates, 1013 were left. The majority of potentially irrelevant literatures were excluded after reviewing the title or abstract. After retrieving 30 full-length manuscripts, finally, 12 articles $[7-9,17,18,24-30]$ of 19 studies were eligible for data extraction and meta-analysis. The flow chart of the studies enrolled in the current study can be found in Fig. 1 . Of the 12 included articles, four articles [8, 17, 25, 30] reported the results according the characteristics of the included participants separately, such as by the sex and BMI. Therefore, during the processes of pooling the results from these articles, these articles were divided into two or more studies.

\section{Study characteristics}

Twelve articles met the inclusion criteria and a total of 21,301 participants were included in the study. In the data analysis process, four articles $[8,16,23,28]$ were divided into two or four studies as the participants were categorized into several groups according their characteristics. Eight studies [7-9, 18, 27-29] addressed the association between sarcopenia and hypertension, and 11 studies [17, 24-26, 30] focused on the association between handgrip strength and hypertension. The sarcopenia was defined by three methods, the European Working Group on Sarcopenia in Older People (EWGSOP) criteria [31], and the Asian Working Group for Sarcopenia (AWGS) criteria. The hypertension was defined as systolic blood pressure (SBP) $>140 \mathrm{mmHg}$ or diastolic blood pressure (DBP) $>90 \mathrm{mmHg}$. Part of the included studies defined handgrip strength following dynamometers according to the protocol from the Institute of Medicine [32]. The included studies were published between 2013 and 2019 and the sample size ranged from 72 to 4771 . The participants' demographic characteristics in the included studies can be found in Supplementary Table 1 and Supplementary Table 2.

Six studies were conducted in China [17, 18, 27, 30], two each in Republic of Korea [8] and Japan [25], and United States [9, 24, 33, 34], one each in Turkey [29], Switzerland [26], Italy [7], and Spain [28]. Most of the studies were cross-sectional studies except two cohort

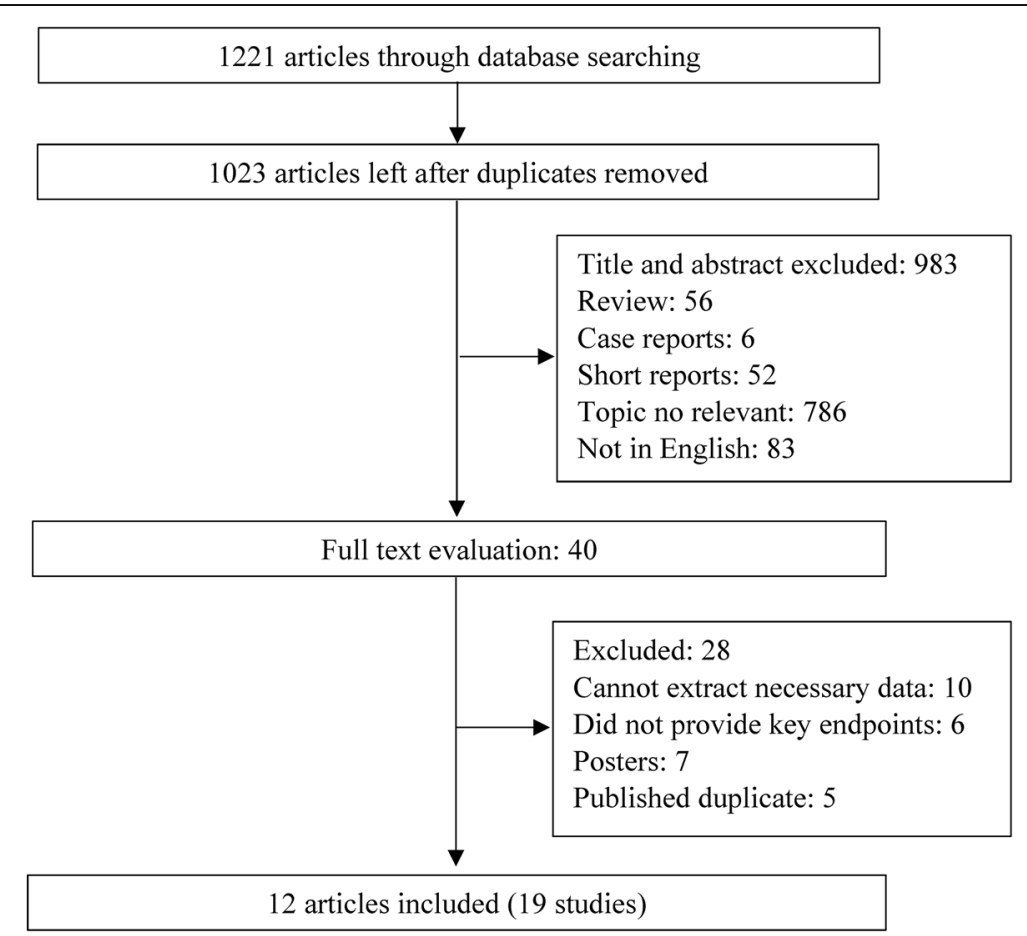

Fig. 1 Flow chart of the study selection 
studies [7, 9]. The characteristics of the included studies and patients were summarized in Tables 1 and 2 .

\section{Quality assessment of studies}

Newcastle-Ottawa Scales for the eligible studies were presented in Supplementary Table 3 and all included studies are found to exhibit an acceptable quality. Four studies were evaluated as 6 stars, 6 studies were 7 stars, and 2 studies were 8 stars.

\section{The association between sarcopenia and hypertension}

All the eight eligible studies have reported the ORs of hypertension, and the ORs ranged from 0.41 to 4.38 . When pooled the ORs together, the summarized OR was 1.29 (95\% CI $=1.00-1.67, P=0.04)$ with a moderate heterogeneity $\left(I^{2}=74 \%\right)$. The detailed information could be found in Fig. 2 and Supplementary Fig. 1.

To explore the sources of heterogeneity, the subgroup analysis was performed by categorizing the studies according to the ethnicity of the participants and the Newcastle-Ottawa Scales than were equal to or more than 7 stars. The Asian group included 4 studies from China and Korea, the Caucasian group included four studies conducted in United States, Italy, Spain, and Turkey. The summarized OR for the Asian group 1.50 (95\% CI $=1.35-1.67, P=0.00$ ) was significantly higher than that of the Caucasian group 1.08 (95\% CI $=0.39-$ 2.97, $P=0.88)$. The heterogeneities for the two subgroups were significantly decreased to $I^{2}=34 \%$ and $I^{2}=$ $40 \%$. When the studies with low quality were removed (Newcastle-Ottawa Scales $<6$ ), the overall OR was 1.53 (95\%CI $=1.37-1.71, P=0.00)$ with lower heterogeneity $\left(I^{2}=2.62 \%\right)$. More data is presented in Figs. 3 and 4 .

\section{The association between handgrip strength and hypertension}

Eleven studies provided data on association between handgrip strength and hypertension, and among them 10 studies reported the odds ratios and 95\% CI. The overall OR and 95\% CI was $0.99(95 \% \mathrm{CI}=0.80-1.23$, $P=0.93)$ with a higher heterogeneity $\left(I^{2}=76 \%\right)$ and significant publication bias $(P<0.01)$. The detailed data can be found in Fig. 5.

As shown in Figs. 6 and 7, in order to explore the sources of heterogeneity and publication bias, the included studies were categorized into two groups by the gender of the participants. For the males, the pooled OR was $1.14(95 \% \mathrm{CI}=0.91-1.43, P=0.27)$ with an acceptable heterogeneity $\left(I^{2}=31 \%\right)$ and without publication bias $(P>0.05)$. The female group did not show any statistically significant difference with an OR as 0.81 $\left(95 \% \mathrm{CI}=0.52-1.26, P=0.34, I^{2}=45 \%\right)$ without publication bias $(P>0.05)$.

Seven studies have reported the $\beta$ value and standard error of the linear regression on hypertension and the pooled $\beta$ value was -1.57 with an SE equal to 1.03 (Fig. 8), and the heterogeneity was $99 \%$. As two studies have provided the data on different body mass indexes, two more subgroup analysis were done, i.e. underweight or normal body mass index group $(\mathrm{OR}=1.04,95 \% \mathrm{CI}=0.81-1.33, P=$ $0.77)$, and overweight or obese body mass index group $(\mathrm{OR}=1.18,95 \% \mathrm{CI}=0.94-1.41, P=0.16)$. The data are presented in Supplementary Fig. 3 and Supplementary Fig. 4.

\section{Publication bias}

Most of the analysis except one has shown potential publication bias among the included trials according to Begg rank correlation analysis and Egger weighted regression

Table 1 Demographic and clinical characteristics of the subjects included in studies that focused on sarcopenia

\begin{tabular}{|c|c|c|c|c|c|c|}
\hline \multirow[t]{2}{*}{ Study included } & \multirow{2}{*}{$\begin{array}{l}\text { No. of } \\
\text { sarcopenia } \\
\text { patients } \\
\text { HTN (-)/ } \\
\text { HTN (+) }\end{array}$} & \multirow{2}{*}{$\begin{array}{l}\text { No. of } \\
\text { non-sarcopenia } \\
\text { patients } \\
\text { HTN (-)/ } \\
\text { HTN (+) }\end{array}$} & \multicolumn{2}{|c|}{ Means \pm SD (mmHg, non-sarcopenia/sarcopenia) } & \multirow{2}{*}{$\begin{array}{l}\% \text { of participants } \\
\text { taking } \\
\text { antihypertensive } \\
\text { drugs }\end{array}$} & \multirow{2}{*}{$\begin{array}{l}\text { Odd ratios } \\
(95 \% \mathrm{Cl})^{c}\end{array}$} \\
\hline & & & SBP & DBP & & \\
\hline Landi et al. [7] & $154 / 126$ & $43 / 28$ & & & & \\
\hline Han et al. ${ }^{a}[8]$ & 2326/1156 & $894 / 544$ & $126.40 \pm 0.50 / 129.30 \pm 0.90$ & $74.30 \pm 0.30 / 75.10 \pm 0.50$ & $33.00 \pm 1.30 / 45.30 \pm 1.90$ & $\begin{array}{l}1.50 \\
(1.23-1.84)\end{array}$ \\
\hline Han et al. ${ }^{b}[8]$ & $594 / 393$ & 1032/771 & $129.90 \pm 0.80 / 131.20 \pm 0.70$ & $76.90 \pm 0.50 / 77.60 \pm .40$ & $52.00 \pm 2.40 / 62.30 \pm 1.90$ & NA \\
\hline Koo et al. [9] & 239/98 & $70 / 41$ & $98.10 \pm 14.8 / 127.9 \pm 17.4$ & $77.8 \pm 11.0 / 77.2 \pm 11.5$ & NA & NA \\
\hline Can et al. [29] & $36 / 4$ & $36 / 12$ & NA & NA & NA & NA \\
\hline Han et al. [24] & $634 / 267$ & $77 / 36$ & NA & NA & NA & NA \\
\hline Montes et al. [28] & $148 / 116$ & $52 / 33$ & NA & NA & NA & NA \\
\hline Xu et al. [18] & 4459/NA & $312 / \mathrm{NA}$ & NA & NA & NA & $\begin{array}{l}1.44 \\
(1.16-1.78)\end{array}$ \\
\hline
\end{tabular}

Abbreviations: HTN Hypertension, SD standard deviation, SBP systolic blood pressure, DBP diastolic blood pressure, $C l$ confidence interval, NA not available

${ }^{a}$, participants whose BMI was less than $25.00 \mathrm{~kg} / \mathrm{m}^{2}$

${ }^{\text {b' }}$, participants whose BMI was equal or more than $25.00 \mathrm{~kg} / \mathrm{m}^{2}$

', Odd ratios stands for hypertension with sarcopenia compared to without sarcopenia 
Table 2 Demographic characteristics of the subjects included in studies that focused on handgrip strength

\begin{tabular}{|c|c|c|c|c|}
\hline Study included & $\begin{array}{l}\text { Sample } \\
\text { size }\end{array}$ & $\begin{array}{l}\text { Handgrip strength } \\
\text { (means } \pm S D \text {, per kg) }\end{array}$ & $\begin{array}{l}\text { Odd ratios }(95 \% \mathrm{Cl}) \\
\text { for High blood pressure }\end{array}$ & $\begin{array}{l}\beta \text { of linear regression } \\
\text { (standard error) }\end{array}$ \\
\hline Mainous, et.al., 2015 [24] & 1469 & $60.8 \pm 1.61^{a} / 71.5 \pm 0.84^{b}$ & NA & $-4.93(0.03)$ \\
\hline Kawamoto, et.al., $2016^{\text {a }}$ [25] & 742 & $33.40 \pm 7.50$ & $0.78(0.66,0.94)$ & NA \\
\hline Kawamoto, et.al., $2016^{\text {b }}$ [25] & 937 & $21.30 \pm 4.10$ & $0.72(0.62,0.83)$ & NA \\
\hline Gubelmann, et.al., $2017^{a}$ [26] & 1891 & NA & $1.23(1.04-1.46)$ & NA \\
\hline Gubelmann, et.al., $2017^{\text {b }}$ [26] & 1577 & NA & $1.01(0.80-1.27)$ & NA \\
\hline Ji, et.al., $2018^{a}$ [17] & 2184 & $41.50 \pm 8.80$ & $1.23(1.04,1.46)$ & $0.21(0.09)$ \\
\hline Ji, et.al., $2018^{b}$ [17] & 2413 & $26.70 \pm 5.70$ & $1.01(0.80,1.27)$ & $0.01(0.12)$ \\
\hline Ji, et.al., $2018^{c}[17]$ & 563 & NA & $1.14(0.83,1.55)$ & $0.13(0.16)$ \\
\hline Ji, et.al., $2018^{d}[17]$ & 1292 & NA & $1.29(1.04,1.59)$ & $0.030(0.17)$ \\
\hline Ji, et.al., $2018 d^{\text {e }}$ [17] & 636 & NA & $0.88(0.58,1.33)$ & $-0.13(0.21)$ \\
\hline Ji, et.al., $2018 d^{f}[17]$ & 1323 & NA & $1.02(0.76,1.38)$ & $0.02(0.15)$ \\
\hline Zhang, et.al., $2019^{a}$ [30] & 515 & $35.94 \pm 19.72$ & $0.55(0.28,1.08)$ & NA \\
\hline Zhang, et.al., $2019^{\text {b }}[30]$ & 637 & $14.45 \pm 10.41$ & $0.19(0.07,0.55)$ & NA \\
\hline
\end{tabular}

Abbreviations: $\mathrm{Cl}$ confidence interval, SD Standard deviation, NA not available

a males

b, females

c, Underweight or normal body mass index of males

d, Underweight or normal body mass index of females

e, Overweight body mass index of males

$f$, Overweight or obese body mass index of females

${ }^{g}$, Odd ratios stands for hypertension with sarcopenia compared to without sarcopenia

analysis ( $P$ value of the analysis was more than 0.05$)$. The detailed potential publication bias of each analysis can be found in Supplementary Table 4.

\section{Discussion}

To the best of our knowledge, the current meta-analysis is the first systematic review and meta-analysis study summarizing the association between sarcopenia and hypertension, and the association of handgrip strength and hypertension. Nineteen studies with 21,301 participants were included, among which eight studies have addressed the association between sarcopenia and hypertension and indicated that sarcopenia was a risk factor for the hypertension. Eleven studies have focused on the association between handgrip strength and hypertension and no association was found by the pooled results.

In the current study, sarcopenia showed an association with hypertension. Several prospective and cross-sectional studies have found a correlation between sarcopenia and hypertension $[8,18]$. In the current study, Asian Working Group for Sarcopenia (AWGS) criteria and the European Working Group on Sarcopenia in Older People

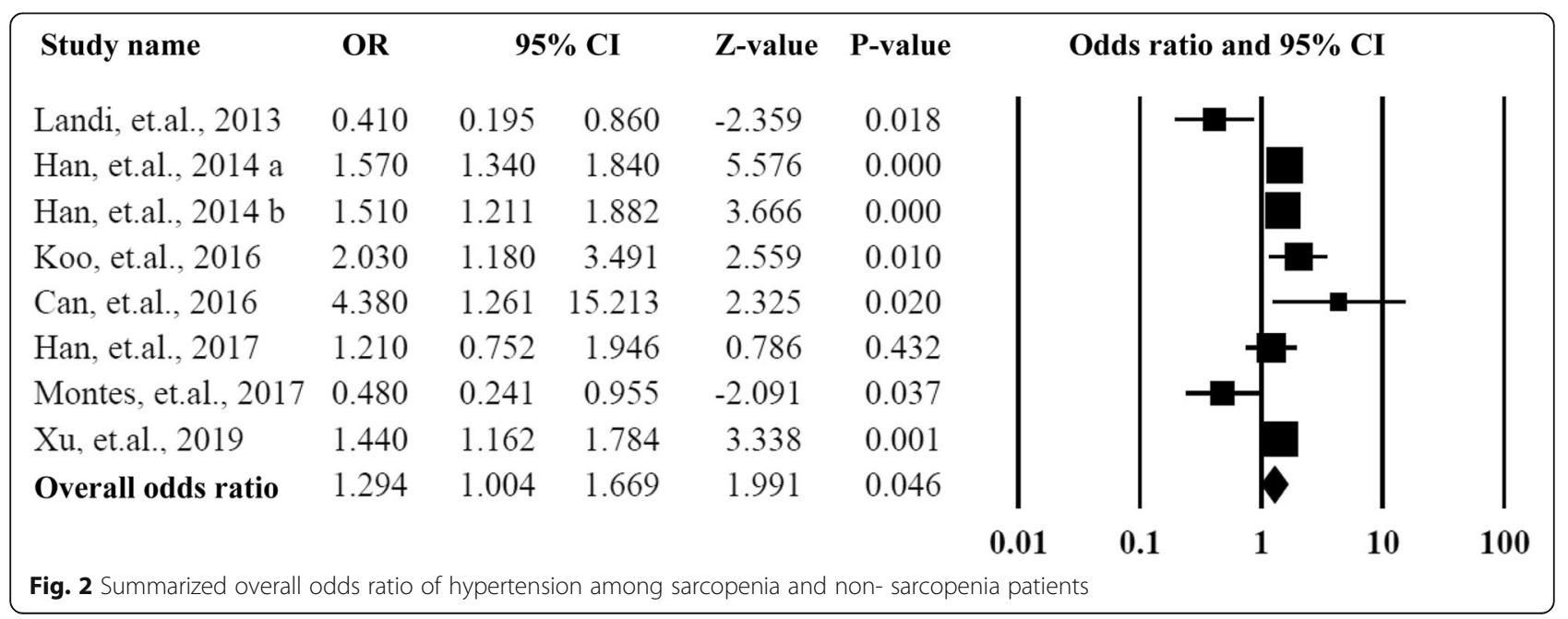




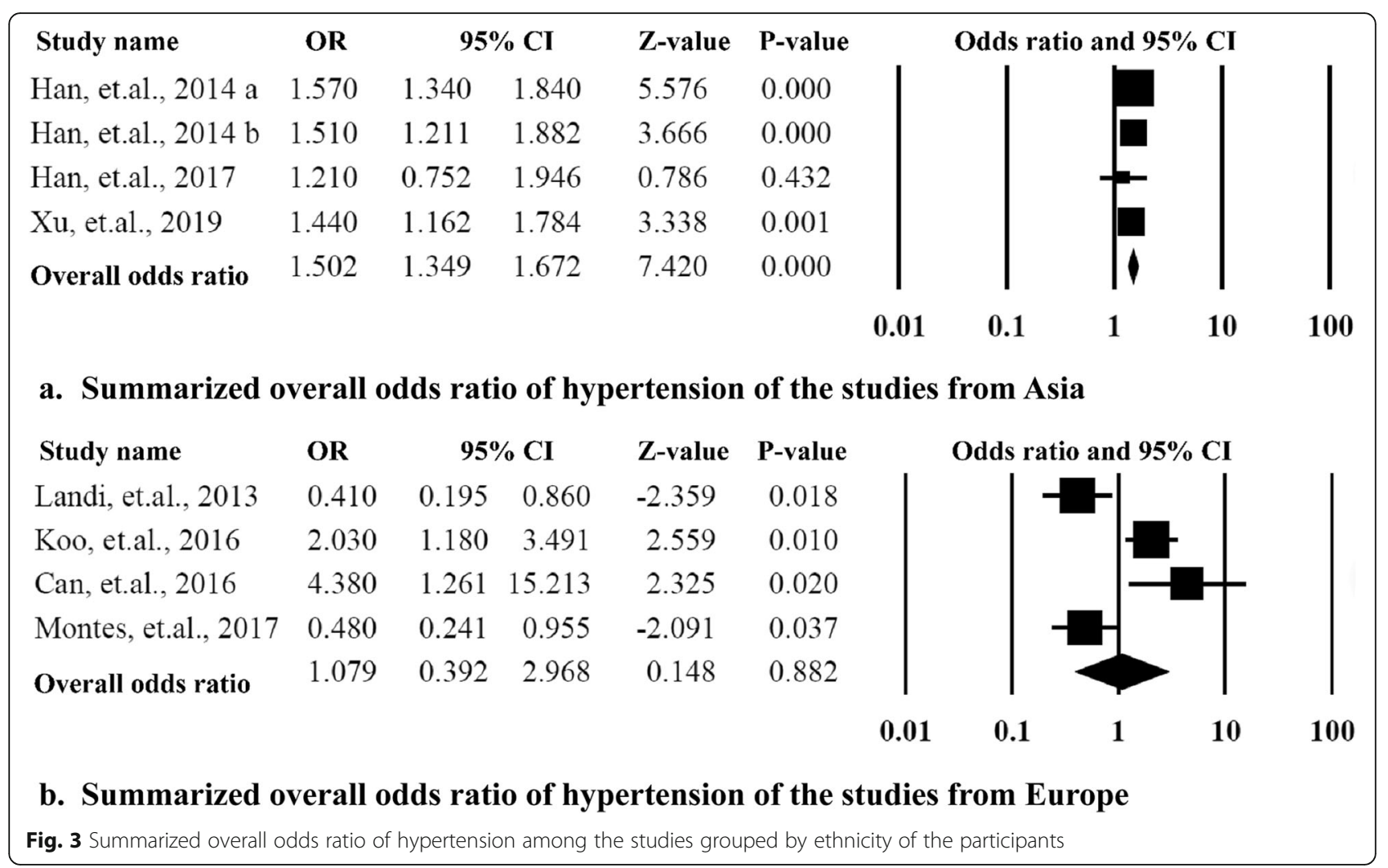

(EWGSOP) criteria were used, which showed significantly different ORs. This might partly explain why Asian groups had a stronger association with hypertension than that of Caucasian group. Many potential mechanisms for sarcopenia have been researched, such as chronic inflammation [35], and its relevant catabolic cytokines remain the most widely accepted mechanism of sarcopenia [35]. It was also reported that chronic inflammation and its relevant catabolic cytokines production is the major risk factor for age-related chronic diseases, such as hypertension [8]. Sarcopenia is not causing hypertension, but it may increase the likelihood of having the other through this shared mechanism and sarcopenia now needs to be recognized in routine clinical settings.

In the current study, handgrip strength was significantly associated with hypertension in both men and women,

\begin{tabular}{|c|c|c|c|c|c|c|c|c|c|}
\hline Study name & $\begin{array}{l}\text { Odds } \\
\text { ratio }\end{array}$ & $\begin{array}{c}\text { Lower } \\
\text { limit }\end{array}$ & $\begin{array}{c}\text { Upper } \\
\text { limit }\end{array}$ & Z-Value & p-Value & \multicolumn{4}{|c|}{ Odds ratio and $95 \% \mathrm{CI}$} \\
\hline Han, et.al., 2014 a & 1.570 & 1.340 & 1.840 & 5.576 & 0.000 & & & & \\
\hline Han, et.al., 2014 b & 1.510 & 1.211 & 1.883 & 3.657 & 0.000 & & & & \\
\hline Koo, et.al., 2016 & 2.030 & 1.180 & 3.492 & 2.559 & 0.011 & & & & \\
\hline Can, et.al., 2016 & 4.380 & 1.261 & 15.219 & 2.324 & 0.020 & & & & \\
\hline Han, et.al., 2017 & 1.210 & 0.751 & 1.949 & 0.784 & 0.433 & & & & \\
\hline Xu, et.al., 2019 & 1.440 & 1.161 & 1.786 & 3.321 & 0.001 & & & & \\
\hline \multirow[t]{2}{*}{ Overall odds ratio } & 1.531 & 1.374 & 1.706 & 7.708 & 0.000 & & & 1 & \\
\hline & & & & & & 0.01 & 0.1 & 10 & 100 \\
\hline
\end{tabular}




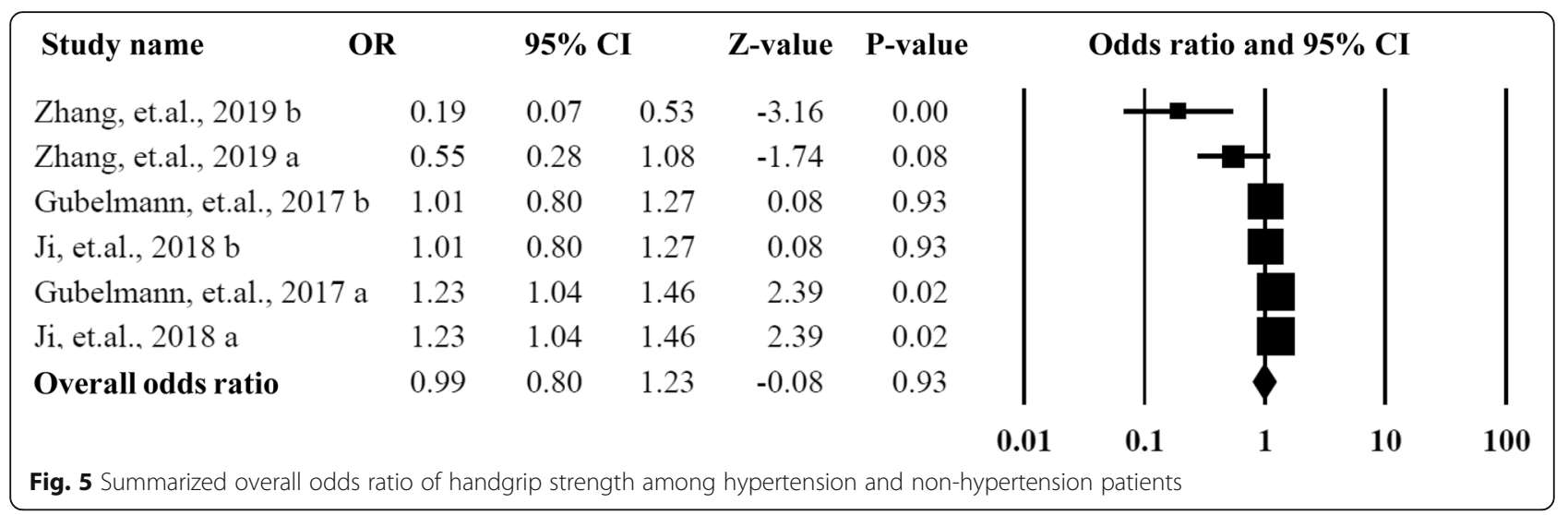

\begin{tabular}{|c|c|c|c|c|c|c|c|c|}
\hline Study name & OR & \multicolumn{2}{|c|}{$95 \%$ CI } & Z-value & P-value & Odds ratio a & $5 \% \mathrm{C}$ & \\
\hline Gubelmann, et.al., 2017 a & 1.23 & 1.04 & 1.46 & 2.39 & 0.02 & & & \\
\hline Ji, et.al., 2018 a & 1.23 & 1.04 & 1.46 & 2.39 & 0.02 & & & \\
\hline Zhang, et.al., 2019 a & 0.55 & 0.28 & 1.08 & -1.74 & 0.08 & & & \\
\hline Overall odds ratio & 1.14 & 0.91 & 1.43 & 1.11 & 0.27 & & & \\
\hline & & & & & 0.01 & 0.1 & 10 & 100 \\
\hline
\end{tabular}

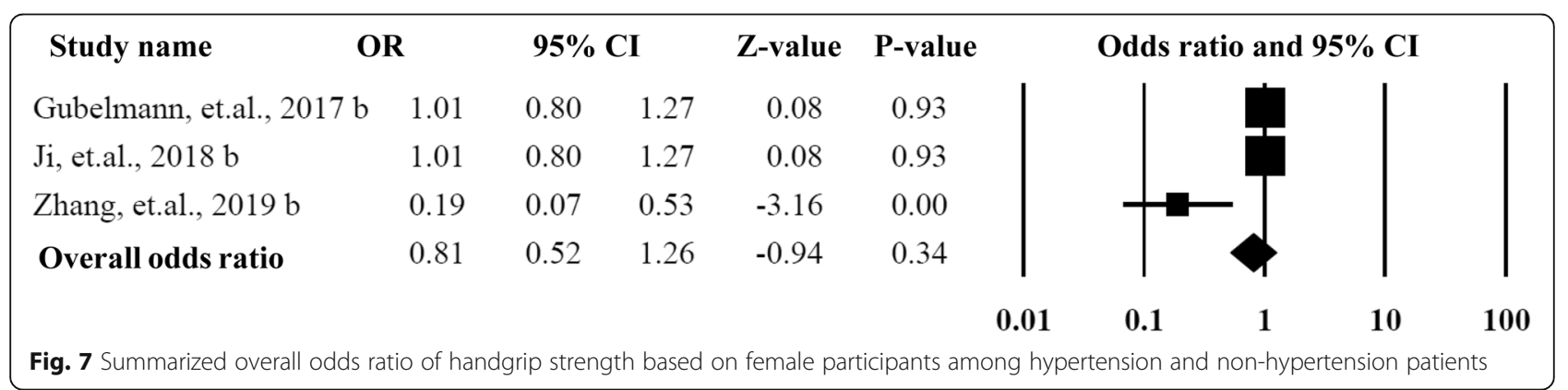

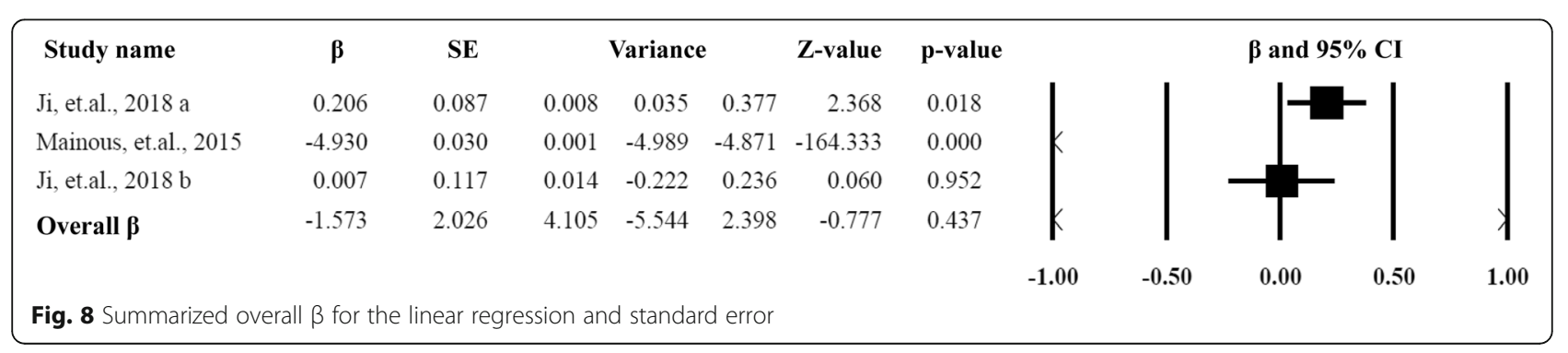


and these results were controversial in various other studies $[25,26]$. However, regular exercise, which has been shown consistently in plenty of studies to improve blood pressure, may eventually improve the mitochondrial function, reduce inflammation, enhance metabolic functions and alleviate sarcopenia symptoms [36].

Frailty is the most problematic expression of population ageing. It is a state of vulnerability to poor resolution of homeostasis and is a consequence of cumulative decline in many physiological systems during a lifetime. The adverse outcomes of frailty might be mediated by sarcopenia, which may be considered the biological substrate for the development of frailty and the related negative health outcomes [37]. Most importantly, it is becoming exceedingly important for us to verify whether preventive strategies focusing on the early detection and treatment of sarcopenia could lead to better survival in the older people.

It is necessary to consider the limitations of the present meta-analysis while interpreting the results. First, the definition of sarcopenia was inconsistent in different studies and the variations in assessment of sarcopenia across studies could have caused methodological limitations and compromised the results. Second, the number of the included studies was limited and the majority of them were from Asian countries. As sarcopenia might be affected by the economic level, medical level, and genetic factors, the associations between sarcopenia, handgrip strength, and hypertension in different countries could be different. Therefore, the result in the current study can only partly annotate the associations. Third, almost all of the studies addressing sarcopenia did not provide the specific breakdown of sarcopenia measurements by gender and age. Due to the limited sample size and information in each study we could not perform more subgroup or sensitivity analyses, e.g. the sensitivity analyses based on the age and sex. Moreover, due to the large disparity in the numbers of participants among the included studies, the conclusions related to the association of sarcopenia with hypertension might be overstated. Four, potential language bias might have existed because our literature searches only considered articles published in English or Chinese.

\section{Conclusions}

In conclusion, our meta-analysis provided pooled results based on 19 studies from eight different countries and summarized a large data set of 21,301 participants. The current study highlighted that sarcopenia was significantly associated with hypertension. In the future, by stratifying patients, efforts must be made to prevent and treat sarcopenia in the older population. At the same time, performing larger sample size studies from different countries in the future may substantially corroborate the conclusive remarks derived from this study.

\section{Supplementary information}

Supplementary information accompanies this paper at https://doi.org/10. 1186/s12877-020-01672-y.

Additional file 1: Supplementary Figure 1. Funnel plot for the overall odds ratio of hypertension among sarcopenia and non- sarcopenia patients. Supplementary Figure 2. Funnel plot for the odds ratio of handgrip strength among hypertension and non-hypertension patients. Supplementary Figure 3. Summarized overall odds ratio of underweight or normal body mass index (BMI) in patients. Supplementary Figure 4. Summarized overall odds ratio of overweight or obese body mass index in patients.

Additional file 2: Supplementary Table 1. Demographic and clinical characteristics of the subjects included in studies that focused on sarcopenia. Supplementary Table 2. Demographic and clinical characteristics of the subjects included in studies that focused on handgrip strength. Supplementary Table 3. Quality assessment of included studies by Newcastle-Ottawa Scale. Supplementary Table 4. Publication bias of summarized outcome.

Additional file 3. Search strategy.

\section{Abbreviations}

ORs: Odd ratios; Cl: Confidence interval; PRISMA: Preferred Reporting Items for Systematic Reviews and Meta-Analyses; NOS: Newcastle-Ottawa Scale; AWGS: Asian Working Group for Sarcopenia; EWGSOP: European Working Group on Sarcopenia in Older People

\section{Acknowledgements}

None.

\section{Authors' contributions}

Study concept: JMC and TTB. Study design: JMC, TTB and FF. Data acquisition: TTB, FF and FKL. Quality control of data and algorithms: JMC and FKL. Data analysis and interpretation: TTB, YR and JAH. Manuscript preparation: TTB and FF. Manuscript editing: TTB and FKL. Manuscript review: JMC and JAH. All authors (TTB, FF, FKL, YR, JAH, JMC) have read and approved the final version of the manuscript.

\section{Funding}

The study was supported by the grant from Shanghai Jiaotong University School of Medicine [TM201805], which had no role in the design of the study and collection, analysis, and interpretation of data and in writing the manuscript.

\section{Availability of data and materials}

Since it is a meta-analysis, all data were extracted from public database, then all data were available.

Ethics approval and consent to participate

Not applicable.

Consent for publication

Not applicable.

Competing interests

The authors declare that they have no competing interests.

Received: 3 January 2020 Accepted: 23 July 2020

Published online: 06 August 2020

References

1. Nations U. World population prospects. New York: United Nations Publications; 2017.

2. Mozaffarian D, Benjamin EJ, Go AS, Arnett DK, Blaha MJ, Cushman M, et al. Heart disease and stroke Statistics-2016 update: a report from the American 
Heart Association. Circulation. 2016;133(4):e38-360. https://doi.org/10.1161/ cir.0000000000000350.

3. Organization WH. Global Health risks: mortality and burden of disease attributable to selected major risks. Geneva: Switzerland World Health Organization; 2009.

4. McLaughlin W, Vachiery JL, Oudiz RJ, Rosenkranz S, Galie N, Barbera JA, et al. Patients with pulmonary arterial hypertension with and without cardiovascular risk factors: results from the AMBITION trial. J Heart Lung Transplant. 2019;38(12):1286-95. https://doi.org/10.1016/j.healun.2019.09.010.

5. Cingolani $\mathrm{OH}$. Cardiovascular risks and organ damage in secondary hypertension. Endocrinol Metab Clin N Am. 2019;48(4):657-66. https://doi. org/10.1016/j.ecl.2019.08.015.

6. Nieman LK. Hypertension and cardiovascular mortality in patients with Cushing syndrome. Endocrinol Metab Clin N Am. 2019;48(4):717-25. https:// doi.org/10.1016/j.ecl.2019.08.005.

7. Landi F, Cruz-Jentoft AJ, Liperoti R, Russo A, Giovannini S, Tosato M, et al. Sarcopenia and mortality risk in frail older persons aged 80 years and older: results from ilSIRENTE study. Age Ageing. 2013;42(2):203-9. https://doi.org/ 10.1093/ageing/afs194.

8. Han K, Park YM, Kwon HS, Ko SH, Lee SH, Yim HW, et al. Sarcopenia as a determinant of blood pressure in older Koreans: findings from the Korea National Health and nutrition examination surveys (KNHANES) 2008-2010. PLoS One. 2014;9(1):e86902. https://doi.org/10.1371/journal.pone.0086902.

9. Koo BK, Kim D, Joo SK, Kim JH, Chang MS, Kim BG, et al. Sarcopenia is an independent risk factor for non-alcoholic steatohepatitis and significant fibrosis. J Hepatol. 2017;66(1):123-31. https://doi.org/10.1016/j.jhep.2016.08. 019.

10. Cruz-Jentoft AJ, Baeyens JP, Bauer JM, Boirie Y, Cederholm T, Landi F, et al. Sarcopenia: European consensus on definition and diagnosis: report of the European working group on sarcopenia in older people. Age Ageing. 2010; 39(4):412-23. https://doi.org/10.1093/ageing/afq034.

11. Woodrow G. Body composition analysis techniques in the aged adult: indications and limitations. Curr Opinion Clin Nutr Metab Care. 2009;12(1):814. https://doi.org/10.1097/MCO.0b013e32831b9c5b.

12. Narici MV, Maffulli N. Sarcopenia: characteristics, mechanisms and functional significance. Br Med Bull. 2010;95:139-59. https://doi.org/10.1093/bmb/ Idq008.

13. Dip RM, Cabrera MA, Prato SF. Association between body composition and stair negotiation ability among individuals $>55$ years of age: a crosssectional study. Clin Interv Aging. 2017;12:1289-96. https://doi.org/10.2147/ cia.s126779.

14. Cawthon PM, Marshall LM, Michael Y, Dam TT, Ensrud KE, Barrett-Connor E, et al. Frailty in older men: prevalence, progression, and relationship with mortality. J Am Geriatr Soc. 2007;55(8):1216-23. https://doi.org/10.1111/j. 1532-5415.2007.01259.x.

15. Landi F, Calvani R, Lorenzi M, Martone AM, Tosato M, Drey M, et al. Serum levels of C-terminal agrin fragment (CAF) are associated with sarcopenia in older multimorbid community-dwellers: results from the iISIRENTE study. Exp Gerontol. 2016;79:31-6. https://doi.org/10.1016/j.exger.2016.03.012

16. Curcio F, Ferro G, Basile C, Liguori I, Parrella P, Pirozzi F, et al. Biomarkers in sarcopenia: a multifactorial approach. Exp Gerontol. 2016;85:1-8. https://doi. org/10.1016/j.exger.2016.09.007.

17. Ji C, Zheng L, Zhang R, Wu Q, Zhao Y. Handgrip strength is positively related to blood pressure and hypertension risk: results from the National Health and nutrition examination survey. Lipids Health Dis. 2018;17(1):86. https://doi.org/10.1186/s12944-018-0734-4

18. Xu HQ, Shi JP, Shen C, Liu Y, Liu JM, Zheng XY. Sarcopenia-related features and factors associated with low muscle mass, weak muscle strength, and reduced function in Chinese rural residents: a cross-sectional study. Arch Osteoporos. 2018;14(1):2. https://doi.org/10.1007/s1 1657-018-0545-2.

19. Liberati A, Altman DG, Tetzlaff J, Mulrow C, Gotzsche PC, loannidis JP, et al. The PRISMA statement for reporting systematic reviews and meta-analyses of studies that evaluate healthcare interventions: explanation and elaboration. BMJ (Clin Res ed.). 2009;339:b2700. https://doi.org/10.1136/bmj. b2700.

20. G W. The Newcastle Ottawa Scale (NOS) for assessing the quality of nonrandomised studies in meta-analysis. England: Paper presented at: Proceedings of the Third Symposium on Systematic Reviews; 2000.

21. Higgins JP, Thompson SG, Deeks JJ, Altman DG. Measuring inconsistency in meta-analyses. BMJ (Clin Res ed). 2003;327(7414):557-60. https://doi.org/10. 1136/bmj.327.7414.557.
22. Begg CB, Mazumdar M. Operating characteristics of a rank correlation test for publication bias. Biometrics. 1994;50(4):1088-101.

23. Egger M, Davey Smith G, Schneider M, Minder C. Bias in meta-analysis detected by a simple, graphical test. BMJ (Clin Res ed.). 1997;315(7109):62934. https://doi.org/10.1136/bmj.315.7109.629.

24. Mainous AG 3rd, Tanner RJ, Anton SD, Jo A. Grip strength as a marker of hypertension and diabetes in healthy weight adults. Am J Prev Med. 2015, 49(6):850-8. https://doi.org/10.1016/j.amepre.2015.05.025.

25. Kawamoto R, Ninomiya D, Kasai Y, Kusunoki T, Ohtsuka N, Kumagi T, et al. Handgrip strength is associated with metabolic syndrome among middleaged and elderly community-dwelling persons. Clin Exp Hypertension. 2016; 38(2):245-51. https://doi.org/10.3109/10641963.2015.1081232.

26. Gubelmann C, Vollenweider P, Marques-Vidal P. Association of grip strength with cardiovascular risk markers. Eur J Prev Cardiol. 2017;24(5):514-21. https://doi.org/10.1177/2047487316680695.

27. Han P, Yu H, Ma Y, Kang L, Fu L, Jia L, et al. The increased risk of sarcopenia in patients with cardiovascular risk factors in suburb-dwelling older Chinese using the AWGS definition. Sci Rep. 2017;7(1):9592. https://doi.org/10.1038/ s41598-017-08488-8.

28. Coto Montes A, Boga JA, Bermejo Millo C, Rubio Gonzalez A, Potes Ochoa $Y$, Vega Naredo I, et al. Potential early biomarkers of sarcopenia among independent older adults. Maturitas. 2017;104:117-22. https://doi.org/10 1016/j.maturitas.2017.08.009.

29. Can B, Kara O, Kizilarslanoglu MC, Arik G, Aycicek GS, Sumer F, et al. Serum markers of inflammation and oxidative stress in sarcopenia. Aging Clin Exp Res. 2017;29(4):745-52. https://doi.org/10.1007/s40520-016-0626-2.

30. Zhang X, Huang L, Peng X, Xie Y, Bao X, Huang J, et al. Association of handgrip strength with hypertension among middle-aged and elderly people in Southern China: A cross-sectional study. Clin Exp Hypertension. 2019:1-7. https://doi.org/10.1080/10641963.2019.1601206.

31. Cruz-Jentoft AJ, Bahat G, Bauer J, Boirie Y, Bruyère O, Cederholm T, et al. Sarcopenia: revised European consensus on definition and diagnosis. Age Ageing. 2019;48(4):601. https://doi.org/10.1093/ageing/afz046.

32. Pate RR, Daniels $S$. Institute of Medicine report on fitness measures and health outcomes in youth. JAMA Pediatr. 2013;167(3):221-2. https://doi.org/ 10.1001/jamapediatrics.2013.1464

33. Pinero F, Mendizabal M, Quiros R, Fauda M, Arufe D, Gonzalez Campana A, et al. Neurological events after liver transplantation: a single-center experience. Transpl Int. 2014;27(12):1244-52. https://doi.org/10.1111/tri. 12404.

34. Kork F, Rimek A, Andert A, Becker NJ, Heidenhain C, Neumann UP, et al. Visual quality assessment of the liver graft by the transplanting surgeon predicts postreperfusion syndrome after liver transplantation: a retrospective cohort study. BMC Anesthesiol. 2018;18(1):29. https://doi.org/10.1186/ s1 2871-018-0493-9.

35. Schrager MA, Metter EJ, Simonsick E, Ble A, Bandinelli S, Lauretani F, et al. Sarcopenic obesity and inflammation in the InCHIANTI study. J Appl Physiol. 2007:102(3):919-25. https://doi.org/10.1152/japplphysiol.00627.2006.

36. Liguori I, Russo G, Aran L, Bulli G, Curcio F, Della-Morte D, et al. Sarcopenia: assessment of disease burden and strategies to improve outcomes. Clin Interv Aging. 2018;13:913-27. https://doi.org/10.2147/cia.s149232.

37. Lackland DT, Voeks JH. Metabolic syndrome and hypertension: regular exercise as part of lifestyle management. Curr Hypertens Rep. 2014;16(11): 492. https://doi.org/10.1007/s11906-014-0492-2.

\section{Publisher's Note}

Springer Nature remains neutral with regard to jurisdictional claims in published maps and institutional affiliations. 